\title{
Blood genotyping for improved outcomes in chronic transfusion patients: current and future perspectives
}

This article was published in the following Dove Press journal:

International Journal of Clinical Transfusion Medicine

4 September 2014

Number of times this article has been viewed

\author{
Jose Mauro Kutner' \\ Mariza Mota' \\ Fabiana Conti' \\ Lilian Castilho ${ }^{1,2}$ \\ 'Hemotherapy and Cell Therapy \\ Department, Hospital Israelita \\ Albert Einstein, São Paulo, SP, Brazil; \\ ${ }^{2}$ Hemocentro Unicamp, Campinas, SP, \\ Brazil
}

\begin{abstract}
Blood transfusions are life sustaining in chronically transfused patients. However, certain complications, such as alloimmunization to red blood cells, can create challenges in the management of those patients. Routine phenotyping of blood recipients and the use of phenotype-matched blood units for transfusion have been useful to lower the occurrence of red cell alloantibodies in chronically transfused individuals. Nevertheless, extensive phenotyping is expensive, laborious, and cannot be performed in certain situations. The molecular understanding of blood groups has enabled the design of assays that may be used to better guide matched red blood cell transfusions. This review summarizes key findings related to red cell alloimmunization, the already identified and potential future benefits of blood group genotyping, and how molecular typing is being incorporated in the blood bank's routine to improve clinical and long-term outcomes in chronically transfused patients.
\end{abstract}

Keywords: blood group genotyping, chronically transfused patients, platelet genotyping, RBC alloimmunization

\section{Introduction}

Red blood cell (RBC) transfusions have been used for decades to treat patients with acute and chronic anemic conditions. Currently, transfusion is one of the most common medical procedures performed in the developed world. ${ }^{1}$ It is indisputable that RBC transfusions help to save lives in diverse acute situations and ameliorate suffering in chronic patients. Because of the recognition that it could also cause major deleterious effects, the practice of donation, processing, testing, storage, and infusion have gone through major changes in the last decades. These improvements have resulted in much safer practices, with some of the risks left only to residual statistical cases. These medical advancements, however, have not resolved all problems.

The challenge involved in the decision to perform a medical procedure, regarding its benefits and risks, varies according to the patient's clinical condition and to his or her short- and long-term prognosis. For patients with chronic conditions, whose treatment inevitably includes RBC transfusions for months or years, it is therefore of benefit to have a programmed approach in order to minimize adverse effects, such as erythrocyte alloimmunization.

Correspondence: Jose Mauro Kutner

Departamento de Hemoterapia e Terapia Celular, Hospital Israelita Albert Einstein, Avenida Albert Einstein, 627/70I, Morumbi, São Paulo, SP,

Brazil, CEP 0565I 901

Tel +55 II 215 I 0444

Fax +55 II 21510446

Email kutner@einstein.br

\section{Blood group genotyping}

The age of genomics has enabled the application of DNA-based molecular methods to transfusion medicine. DNA technology has led to the understanding of the molecular basis of many common blood group antigens, and now most of the genes governing 
blood group systems have been cloned and sequenced. ${ }^{2}$ There are many molecular events that give rise to blood group antigens and phenotypes. However, the majority of genetically defined blood group antigens are the consequence of a single-nucleotide polymorphism (SNP). This knowledge allows the use of deoxyribonucleic acid (DNA)-based assays to detect specific blood group SNPs which can be used to overcome the limitations of hemagglutination assays. ${ }^{3}$

Several assays for blood group genotyping have been developed to predict the blood group antigen profile of an individual. They include polymerase chain reaction (PCR)restricted fragment length polymorphism, allele-specific PCR as single or multiplex assays, sequence-based assays, real-time quantitative PCR, and DNA array methods.

DNA analysis is presently available for clinical use in various situations. It is a useful tool to help identify rare $\mathrm{RhD}$ blood group antigen variants; type the patients' RBCs when they have received multiple transfusions or have positive direct antiglobulin tests; determine RHD zygosity; genotype donors for certain antigens (eg, Dombrock) that are hard to ascertain because of antisera unavailability or weak potency; determine $R H D$ type fetuses (eg, a fetus carried by an RhD negative mother) without using invasive procedures; determine which phenotypically antigen-negative patients can receive antigen-positive $\mathrm{RBCs}$; type donors for antibody identification panels; type patients who have an antigen that is expressed weakly on RBCs; and resolve $\mathrm{A}, \mathrm{B}$, and $\mathrm{RhD}$ discrepancies. More recently, it has been used in donors to mass screen for antigen-negative individuals. Table 1 summarizes the main applications of blood group genotyping in patients and donors. ${ }^{5,6}$

A molecular understanding of blood groups has enabled the design of simple assays that may be used to facilitate the provision of blood to patients who require antigen-matched $\mathrm{RBC}$, both by phenotyping the patient to determine his or her requirements, and by phenotyping red cell units. ${ }^{4}$ It is unclear how long it will be before all donors are genotyped rather than phenotyped. ${ }^{5,6}$ The outcome will obviously depend a great deal on cost-benefit analysis and the availability of high-throughput platforms.

The development of high-throughput genotyping platforms that utilize microarray and chip technologies offers the opportunity to perform large-scale testing on numerous antigens simultaneously, allowing an accurate selection of donor units to facilitate matching of donor RBCs to the recipient's blood type. This creates the opportunity to extend routine antigen matching between donors and recipients to
Table I Applications of blood group genotyping

\section{Patients}

- Type the patients' own RBCs when they have received multiple transfusions or have positive direct antiglobulin tests

- Help identify rare RhD variants

- Determine which phenotypically antigen-negative patients can receive antigen-positive RBCs

- Type patients who have an antigen that is expressed weakly on RBCs

- Resolve A, B, and RhD discrepancies

- RHD type fetuses (eg, one carried by an RhD negative mother) without using invasive procedures

- Determine RHD zygosity

- Distinguish an alloantibody from an autoantibody

- Type patients who have received allogeneic stem cell transplant

Donors

- Genotype donors for certain antigens (eg, Dombrock) that are hard to ascertain because of antisera unavailability or weak potency

- Type donors for antibody identification panels

- Mass screen for antigen-negative individuals

- Find rare donors

- Increase the inventory of antigen-negative units

prevent alloimmunization to $\mathrm{RBC}$ antigens. ${ }^{7}$ Commercial kits are available, and hopefully will be approved by regulatory agencies worldwide soon.

By testing patients and donors, it is possible to provide more accurately matched blood, thus preventing additional alloimmunization. A study by Klapper et $\mathrm{al}^{8}$ using the human erythrocyte antigen BeadChip ${ }^{\text {TM }}$ DNA analysis (BioArray ${ }^{\text {TM}}$; Immucor, Norcross, GA, USA) and a Web-based inventory management system to model donor-recipient matching, showed that even with a limited donor pool, matching for Rh, Kell, Duffy, Kidd, and MNS systems could be achieved in at least $50 \%$ of the cases. The ability to test patients and a large number of donors simultaneously for several antigens, together with computer analysis and interpretation of data, ${ }^{8,9}$ makes it feasible to facilitate the matching of RBC components to recipients' blood type, and increases the available inventory of donor units to chronically transfused patients. Klapper et $\mathrm{al}^{8}$ concluded that if patients and donors are extensively DNA typed, it is feasible to provide units for transfusion that are better matched than the current standard of practice allows.

\section{The chronically transfused patient}

Certain diseases, either congenital or acquired, may require frequent RBC transfusions. Patients with sickle cell anemia (SCA), thalassemia, and myelodysplastic syndromes (MDS) are the most frequently reported in medical literature regarding red cell antibody formation. ${ }^{23,26,31}$ 


\section{SCA}

The HbS gene has an ubiquitous distribution and affects mainly subjects of African descent. ${ }^{10} \mathrm{RBC}$ transfusions optimize oxygen delivery, reduce the RBC sickling predisposition by diluting the patient's abnormal cells, and temporarily suppress the production of red cells containing HbS. ${ }^{11}$ Patients with SCA have a predisposition to form alloantibodies. ${ }^{12}$ Possible causes include antigenic differences between donors and recipients, a chronic inflammatory state with increased levels of C-reactive protein, inflammatory cytokines, and hyper-reactive macrophages. ${ }^{12-14}$ The occurrence of alloimmunization to RBC in patients with SCA ranges from $18 \%$ to $47 \%$ at a rate of 1.7 to 3.8 alloantibodies formed per 100 transfused units. ${ }^{15}$ Lower rates of alloimmunization are found in countries where donors and recipients have a lower disparity in red cell antigenic profiles. ${ }^{16}$ Most of the alloantibodies have Rh and Kell blood group specificities. ${ }^{12}$ It is therefore expected that a better donor-recipient antigen match, may result in a lower rate of alloimmunization.

\section{Thalassemia}

In thalassemia, a congenital hemolytic disorder, the reduced rate of synthesis of one or more of the globin chains leads to imbalanced globin-chain synthesis, defective hemoglobin production, and damage to the red cells resulting from the effects of the globin subunits that are produced in relative excess. ${ }^{17}$ The alloimmunization rate can range from $5.2 \%$ up to $30 \% .{ }^{17-21}$ The more homogeneous the population, the lower the sensitization rates, ${ }^{12}$ reinforcing the fact that better matched $\mathrm{RBC}$ transfusions are key to reducing alloimmunization rates. Expanding the compatibility of RBC to other blood groups beyond $\mathrm{ABO}$ and $\mathrm{RhD}$, reduces the alloimmunization rates in thalassemic patients. By extending antigen compatibility to $\mathrm{Rh}$ and $\mathrm{K}$ antigens, Michail-Merianou et $\mathrm{al}^{22}$ reported a reduction in alloimmunization rate from $23.4 \%$ to $14.3 \%$. Likewise, Spanos et $\mathrm{al}^{23}$ have reported a reduction from $23.5 \%$ to $3.7 \%$.

\section{MDS}

MDS are a group of heterogeneous clonal disorders characterized by ineffective hematopoiesis and peripheral cytopenias. ${ }^{24}$ Many patients with MDS evolve with symptomatic anemia during the course of the disease. Although some patients may respond to drug therapy such as recombinant erythropoietin, azacitidine, and lenalidomide, frequent RBC transfusions remain an important resource in their treatment. ${ }^{24,25}$ Sanz et $\mathrm{al}^{26}$ reported a $15 \%$ alloimmunization rate against red cell antigens in MDS patients, mostly involving Rh and
Kell antigens. These authors have observed that the incidence of RBC alloimmunization increased with the number of transfusions and did not reach a plateau until 130 units were transfused. Twenty-one percent of the units given to the patients in the study needed extended compatibility beyond routine $\mathrm{ABO} / \mathrm{RhD}$, and $10 \%$ of all transfusion episodes required the performance of sophisticated immunohematologic studies to resolve complex cases.

\section{Alloimmunization to RBC antigens}

Alloantibody formation to RBC antigens is a major complication associated with $\mathrm{RBC}$ transfusions, especially in chronically transfused patients. ${ }^{27}$ Alloantibodies restrict the number of compatible units, may complicate transfusions, and can delay the procedure when compatible products are not readily available. ${ }^{28,29}$ Alloimmunized patients also have an increased risk of delayed hemolytic transfusion reactions and autoantibody formation, and those who develop an alloantibody have a greater chance of developing others with future transfusions..$^{30}$ Even non-chronically transfused patients who become alloimmunized are 20 times more likely to form additional antibodies after one or more repeated transfusion events. ${ }^{31-33}$

The rate of RBC alloimmunization in transfused patients shows great variation and is reported to range from $2.6 \%$ up to $60 \%$, depending on the patient population and methodology used. ${ }^{34}$ The risk of alloimmunization may be influenced by disparities between donor and recipient antigenic profiles, by individual and ethnic differences, and also by other factors such as dose, mode of exposure, immunogenicity of the antigen as well as genetic, acquired patient-related factors and clinical conditions. ${ }^{10,35,36}$ It has also been shown that the number of transfusions plays an important role in RBC alloimmunization, and that immune-compromised patients have a lower risk of developing red cell antibodies. ${ }^{37}$

Because of alloimmunization, much time and effort are spent in detecting and identifying blood group antibodies. Next to $\mathrm{ABO}$, the most clinically significant antibodies are those in Rh, Kell, Duffy, and Kidd blood group systems. ${ }^{38}$

Chronically transfused patients represent a special class of alloimmunized individuals, for whom the development of multiple antibodies may ultimately lead to death. In these patients, alloimmunization is of considerable medical importance. ${ }^{26}$

\section{Alloimmunization to platelets}

Platelets contain glycoproteins in their membrane that comprise the human platelet-specific antigen system (HPA). 
This system has a high level of polymorphism, and alloantibodies can be formed during pregnancy or after transfusions, leading to complications like neonatal alloimmune thrombocytopenia, post-transfusion purpura, and refractoriness to platelet transfusions. ${ }^{39,40}$ The alloimmunization rate against HPA depends largely on antigen frequencies, which vary considerably according to the ethnic background. ${ }^{40}$

\section{RBC alloimmunization and chronic transfusions}

Blood group antigens are polymorphisms of proteins and carbohydrates on the outside surface of the RBC, and are defined by serum alloantibodies produced in response to an immunizing event such as transfusion or pregnancy. ${ }^{42}$ There are more than 340 blood group antigens recognized by the International Society of Blood Transfusion (ISBT), 293 of which have been assigned to 34 blood group systems. ${ }^{41}$ Most blood group antigens are inherited traits controlled by a single gene or cluster of two or three closely linked homologous genes. ${ }^{42}$ Antibodies to these antigens cause clinical problems in transfusion incompatibility, maternal-fetal incompatibility, and autoimmune hemolytic anemia. ${ }^{43}$

The provision of antigen-negative blood forms the basis for safe blood transfusion by minimizing the risk of adverse transfusion reactions and alloimmunization. ${ }^{8}$ Some authors have recommended applying extended RBC typing and matching for chronically transfused patients, as this approach has been shown to reduce rates of alloimmunization. ${ }^{44-46}$ Vichinsky ${ }^{47}$ showed a reduction in alloimmunization rates among SCA patients from $3 \%$ to $0.5 \%$ with transfusion of phenotypically matched blood. Although the use of extended phenotyping reduces the rate of alloimmunization, and this practice is advocated by some groups, ${ }^{29-33}$ it has not been widely implemented, and the majority of blood banks do not routinely perform it. ${ }^{30}$ Among the reasons for lack of broadscale implementation are the cost of extended antigen matching when compared to standard red cell typing, and the length of time needed to perform the procedure, which may be more challenging in situations when urgent transfusions are needed. ${ }^{44}$

Programs to prevent alloimmunization to RBC antigens have been designed and implemented to provide antigen-matched RBC transfusions to patients who are alloimmunized and/or in need of chronic transfusion support. ${ }^{31-34}$ Although transfusion services establish protocols to reduce alloimmunization risk, there is no consensus about the ideal protocols that should be used. These protocols range from providing limited phenotype- matched RBCs for only $\mathrm{Rh}(\mathrm{D}, \mathrm{C}$, and $\mathrm{E})$ and $\mathrm{K}$ antigens to transfusing extended phenotype-matched RBCs for Rh (C, c, E, and e), K, S, Fy ${ }^{\mathrm{a}}$, and $\mathrm{Jk}^{\mathrm{a}}$ antigens. ${ }^{48}$ In the authors' institution, (Hospital Israelita Albert EinsteinHIAE) matching for chronically transfused patients such as patients with SCA, thalassemia, MDS, and warm autoimmune hemolytic anemia includes, in addition to $\mathrm{ABO}$, phenotyping for $\mathrm{Rh}\left(\mathrm{C}, \mathrm{c}, \mathrm{E}\right.$, and e), $\mathrm{K}, \mathrm{Fy}^{\mathrm{a}}, \mathrm{Fy}^{\mathrm{b}}, \mathrm{Jk}^{\mathrm{a}}, \mathrm{Jk}^{\mathrm{b}}$, and $\mathrm{S}$, a procedure developed to prevent alloimmunization to these RBC antigens, and as part of the antibody identification process.

RBC phenotyping is essential to confirm the identity of suspected alloantibodies and to facilitate the identification of antibodies that may be formed in the future. Accurate antigen typing of transfused patients can be a difficult task due to the presence of donor RBCs in patients' circulation. Thus, in these patients, phenotyping can be time consuming and difficult to interpret. It is also complicated to type cells when a patient's RBCs yield a positive direct antiglobulin test, and no direct agglutinating antibody is available. ${ }^{49}$

\section{Blood group genotyping in chronically transfused patients}

In 1999, Legler et $\mathrm{al}^{50}$ showed that in chronically transfused patients, molecular typing yielded Rh phenotypes that differed from the serological types in seven of 27 patients. In 2002, Castilho et al showed that genotypes differed from the assumed phenotypes in six of 40 transfused patients with $\mathrm{SCA}^{51}$ and in nine of ten alloimmunized thalassemic patients. $^{52}$ In 2013, Bakanay et al $^{53}$ showed that 19 out of 37 multi-transfused patients had discrepancies between genotyping and phenotyping results in a total of 25 alleles. The discovery of these discrepancies aided in the identification of alloantibodies and the selection of the correct antigen-matched products for those patients. The patients who then were switched to the correct antigen-matched RBCs had improved RBC survival with diminished frequency of transfusions. These studies ${ }^{50-53}$ highlighted the use of genotyping for patients with SCA and with thalassemia for antigen-matched $\mathrm{RBC}$ transfusions, which resulted in improved patient care. Over the past ten years, several other authors have demonstrated that blood group genotyping of transfusion-dependent patients is useful in preventing and identifying alloimmunization and providing appropriate antigen-matched products, and there is now a consensus that molecular blood group phenotype prediction is superior to serological blood group phenotype determination in chronically transfused patients. ${ }^{54-56}$ 
The clinical benefits for transfusion-dependent populations are tangible: better-matched blood decreases the risk of hemolytic transfusion reactions, especially delayed transfusion reactions to existing alloantibodies, and prevents new instances of alloimmunization. ${ }^{52}$ Also, the use of better-matched blood units can reduce transfusion requirements, decreasing the risk of other adverse reactions like transfusion-related acute lung injury and potential exposure to infectious diseases. ${ }^{54-56}$ In summary, matching at DNA level can improve safety and efficacy for chronically transfused patients. Genotyping can also help decrease the costs, in terms of both time and money, associated with complex serological workups and higher number of transfusions.

However, it is important to remember that, regardless of the test protocols used, genotyping can only predict a blood type. In rare situations, a genotype determination will not correlate with antigen expression on RBCs, and appropriate assays are necessary to detect nucleotide changes that alter the predicted phenotype. Given the large number of genetic events that are already known to silence or weaken expression of antigens encoded by an allele, it will be a long time before all relevant nucleotide changes are revealed for all blood group systems in all ethnic groups. For those reasons, care must be taken when using molecular methods in antibody investigations, because the serological situation may involve the inheritance of a null allele, a hybrid gene, or a new variant..$^{57-60}$

In this context, the analysis of blood group variants in a specific population is very important to predict a red cell phenotype using molecular methods. The more we learn about frequency and molecular backgrounds of such variants in different populations, the more accurate will be the genotype/ phenotype results. For example, more work needs to be done to show that molecular methods can recognize variants seen primarily in African populations. Two common examples are the GATA-promoter polymorphism, which silences the $F Y^{*} B$ gene, ${ }^{58}$ and two different $G Y P B$ alleles $\left(G Y P B^{*} P 2\right.$ and $G Y P B^{*} N Y$ ) that silence the expression of the $\mathrm{S}$ antigen. ${ }^{59}$

The S-s- phenotype, with rare exceptions, is found only in individuals of African descent and may be associated with the absence or weak expression of the high-frequency $U$ antigen. S-s- individuals can develop anti-U, which is known to cause decreased survival of transfused antigen-positive RBCs. Therefore, it is very important to predict those phenotypes correctly. ${ }^{59,60}$

Ongoing advances in the automation of SNP and DNA sequence analysis and the success of sequencing the human genome have revealed that the potential for large scale genotyping has already been achieved. ${ }^{61}$ It should soon be possible to analyze major and many minor blood group alleles on a single synthetic chip. Indeed, it will be possible to test for many genetically defined conditions.

As automated procedures attain higher and faster throughput at lower cost, blood group genotyping is likely to become more widespread. As transfusion-dependent patients with SCA, thalassemias, and MDS frequently become alloimmunized, blood group genotyping can contribute substantially to the safety of blood transfusion in these recipients. ${ }^{51-55}$ Therefore, we believe that for chronically transfused patients, DNA technology may be used in a transfusion service in the next few years to replace hemagglutination.

\section{Platelet genotyping}

Many advances observed in blood group genotyping may be extended for platelet transfusions in sensitized patients. Platelet phenotyping can be performed by serological techniques like the modified antigen capture enzyme-linked immunosorbent assay, ${ }^{62}$ monoclonal antibody-specific immobilization of platelet antigens, ${ }^{63}$ and mixed passive hemagglutination.$^{64}$ However, these methods are complex and time-consuming.

Molecular HPA typing has been increasingly used to deal with platelet disorders like platelet refractoriness, neonatal thrombocytopenia, and post-transfusion purpura. The initial PCR-based methods are giving way to large throughput genotyping technologies which, as with RBC antigens, are faster and more accurate. Microarray platforms have also been implemented for use with platelet genotyping. ${ }^{65}$

\section{Incorporating molecular typing in the routine of blood banks}

Two recently published papers have shown the feasibility of routinely applying molecular immunohematology techniques in hospital transfusion services. ${ }^{66,67}$ This is important, as many of the applications of RBC genotyping have been applied mainly to mass screening in blood centers. ${ }^{8,68}$

Shafi et $a^{66}$ described the implementation of routine molecular testing as a strategy to maximize efficient use of blood units in a large tertiary care hospital in Los Angeles, USA. A significant portion of the blood donations were typed using molecular methods, already comprising a donor database of over 10,000 genotyped donors. After using matched blood at molecular level, no novel antibodies were found in a group of previously sensitized patients. All patients with warm or cold reacting autoantibodies, those with antibodies that could not be identified, and patients with SCA were 
molecular typed. Most of the molecularly matched transfused units originated from the hospital's own inventory.

Sapatnekar and Figueroa, ${ }^{67}$ also in a large hospital blood bank in Cleveland, USA, described how they apply pretransfusion molecular typing not only in chronically transfused patients, but also in patients with autoantibodies, multiple antibodies, in situations where no antigen specific antibody was available for testing, and to solve laboratory discrepancies. Up to $4.8 \%$ of patients' antibody screens were positive, and $56 \%$ of the patients for whom extended phenotyping was indicated could not be typed serologically because of recent transfusion or positive direct antiglobulin test (DAT). An algorithm was developed in order to identify which samples should proceed to molecular testing. Staff training and genotyping of donors and patients on a regular basis was shown to be feasible.

In the authors' experience, in the blood bank of a 650 bed tertiary care hospital in Brazil, (HIAE) it is routine to perform blood group genotyping in selected, chronically transfused patients. This practice has improved the ability to find antigen-matched components for transfusion support and has been of benefit to patients, as observed by the lack of newly developed alloantibodies in previously alloimmunized patients and as assessed by increased hemoglobin levels and diminished frequency of transfusions (Kutner et al, unpublished data, 2013).

\section{Conclusion}

Genotyping offers many advantages over serological testing of recipients' blood, with the primary benefit of predicting the blood group phenotype in situations where phenotyping cannot be performed serologically. Molecular testing is a rapidly advancing field that offers tremendous potential in transfusion medicine, has been successfully implemented in immunohematology laboratories, and is proving to be a powerful tool, with potential advantages for identifying rare blood and finding better antigen matches for chronically transfused patients. As many adverse effects of blood transfusions have been solved or minimized, making it a therapeutic tool safer than ever, molecular typing heralds a new era for the prevention of alloantibody formation. With the advance of next-generation sequencing, it will be possible to obtain even more accurate blood group phenotype predictions in both blood donors and patients, thus allowing patient-specific transfusion therapy. ${ }^{69}$ If a situation can be attained where all donors and patients are fully genotyped, it should be possible to safely allocate blood for transfusion without further compatibility testing. This would change current practice fundamentally by removing the need for patient testing in hospital transfusion laboratories. All matching could be computerized. Chronically transfused patients would therefore be able to take the greatest advantage of these new developments.

\section{Disclosure}

The authors report no conflicts of interest in this work.

\section{References}

1. Szczepiorkowski ZM, Dunbar NM. Transfusion guidelines: when to transfuse. Hematology Am Soc Hematol Educ Program. 2013;2013:638-644.

2. Avent ND. Large-scale blood group genotyping: clinical implications. Br J Haematol. 2008;144(1):3-13.

3. Van der Schoot CE. Molecular diagnostics in immunohaematology. Vox Sang. 2004;87(S2):189-192.

4. Rujirojindakul P, Flegel WA. Applying molecular immunohaematology to regularly transfused thalassaemic patients in Thailand. Blood Transfus. 2014;12(1):28-35.

5. Westhoff CM. Molecular testing for transfusion medicine. Curr Opin Hematol. 2006;13(6):471-475.

6. Hillyer CD, Shaz BH, Winkler AM, Reid M. Integrating molecular technologies for red blood cell typing and compatibility testing into blood centers and transfusion services. Transfus Med Rev. 2008;22(2):117-132.

7. Hashmi G, ShariffT, Seul M, et al. A flexible array format for large-scale, rapid blood group DNA typing. Transfusion. 2005;45(5):680-688.

8. Klapper E, Zhang Y, Figueroa P, et al. Toward extended phenotype matching: a new operational paradigm for the transfusion service. Transfusion. 2010;50(3):536-546.

9. Ribeiro KR, Guarnieri MH, da Costa DC, Costa FF, Pellegrino J Jr, Castilho L. DNA array analysis for red blood cell antigens facilitates the transfusion support with antigen-matched blood in patients with sickle cell disease. Vox Sang. 2009;97(2):147-152.

10. Badjie KS, Tauscher CD, van Buskirk C, et al. Red blood cell phenotype matching for various ethnic groups. Immunohematology. 2011;27(1): 12-19.

11. Natakunda B. Red blood alloimmunization and antigen matching in sickle cell disease - the African perspective. ISBT Sci Ser. 2012;7(1): 129-133.

12. Chou ST, Westhoff CM. The role of molecular immunohematology in sickle cell disease. Transfus Apher Sci. 2011;44(1):73-79.

13. Matteocci A, Pierelli L. Red blood cell alloimmunization in sickle cell disease and thalassaemia: current status, future perspectives and potential role of molecular typing. Vox Sang. 2014;106(3):197-208.

14. Noizat-Pirenne F. Relevance of alloimmunization in haemolytic transfusion reaction in sickle cell disease. Transfus Clin Biol. 2012;19(3): 132-138.

15. Campbell-Lee S. Red blood cell alloimmunization in sickle cell anemia: more questions than answers? Transfusion. 2012;52(2):214-216.

16. Lasalle-Williams M, Nuss R, Le T, et al. Extended red blood cell antigen matching for transfusions in sickle cell disease: a review of a 14-year experience from a single center (CME). Transfusion. 2011;51(8):1732-1739.

17. Weatherall DJ. Disorders of globin synthesis: the thalassemias. In: Lichtman MA, Kipps TJ, Daushansky K, et al, editors. Williams Hematology. 8th ed. New York: McGraw Hill; 2006:829-871.

18. Sirchia G, Zanella A, Parravicini A, Morelati F, Rebulla P, Masera G. Red cell alloantibodies in thalassemia major. Results of an Italian cooperative study. Transfusion. 1985;25(2):110-112.

19. Ameen R, Al-Shemmari S, Al-Humood S, Chowdhury RI, Al-Eyaadi O, Al-Bashir A. RBC alloimmunization and autoimmunization among transfusion-dependent Arab thalassemia patients. Transfusion. 2003;43(11):1604-1610. 
20. Azarkeivan A., Ansari S., Ahmadi M.H. et al. Blood Transfusion and Allommunization in patients with thalassemia: Multicenter Study Pediatr Hematol Oncol. 2011;28(6):479-485.

21. Cheng CK, Lee CK, Lin CK. Clinically significant red blood cell antibodies in chronically transfused patients: a survey of Chinese thalassemia major patients and literature review. Transfusion. 2012;52(10):2220-2224.

22. Michail-Merianou V, Pamphili-Panousopoulou L, Piperi-Lowes L, Pelegrinis E, Karaklis A. Alloimmunization to red cell antigens in thalassemia: comparative study of usual versus better-match transfusion programmes. Vox Sang. 1987;52(1-2):95-98.

23. Spanos T, Karageorga M, Ladis V, Peristeri J, Hatzliami A, Kattamis C. Red cell alloantibodies in patients with thalassemia. Vox Sang 1990;58(1):50-55.

24. Tefferi A, Vardiman JW. Myelodysplastic syndromes. $N$ Engl J Med. 2009;361(19):1872-1885.

25. Stone RM. How I treat patients with myelodysplastic syndromes. Blood. 2009;113(25):6296-6303.

26. Sanz C, Nomdedeu M, Belkaid M, Martinez I, Nomdedeu B, Pereira A. Red blood cell alloimmunization in transfused patients with myelodysplastic syndrome or chronic myelomonocytic leukemia. Transfusion. 2013;53(4):710-715.

27. Chou ST, Liem RI, Thompson AA. Challenges of alloimmunization in patients with haemoglobinopathies. Br J Haematol. 2012;159(4):394-404.

28. Stainsby D, Jones H, Asher D, et al; SHOT Steering Group. Serious hazards of transfusion: a decade of hemovigilance in the UK. Transfus Med Rev. 2006;20(4):273-282.

29. Vamvakas EC, Blajchman MA. Blood still kills: six strategies to further reduce allogeneic blood transfusion-related mortality. Transfus Med Rev. 2010;24(2):77-124.

30. Zimring JC, Welniak L, Semple JW, et al. Current problems and future directions of transfusion-induced alloimmunization: summary of an NHLBI working group. Transfusion. 2011;51(2):435-441.

31. Cox JV, Steane E, Cunningham G, Frenkel EP. Risk of alloimmunization and delayed hemolytic transfusion reactions in patients with sickle cell disease. Arch Intern Med. 1988;148(11):2485-2489.

32. Schonewille H, Haak HL, van Zijl AM. Alloimmunization after blood transfusion in patients with hematologic and oncologic diseases. Transfusion. 1999;39(7):763-771.

33. Schonewille H, van de Watering LM, Brand A. Additional red blood cell alloantibodies after blood transfusions in a nonhematologic patient cohort: is it time to take precautionary measures? Transfusion 2006;46(4):630-635

34. Natukunda B, Brand A, Schonewille H. Red blood cell alloimmunization from an African perspective. Curr Opin Hematol. 2010;17(6): 565-570.

35. Bao W, Yu J, Heck S, Yazdanbakhsh K. Regulatory T-cell status in red cell alloimmunized responder and non responder mice. Blood. 2009;113(22):5624-5627.

36. Bao W, Zhong H, Li X, et al. Immune regulation in chronically transfused allo-antibody responder and nonresponder patients with sickle cell disease and $\beta$-thalassemia major. Am J Hematol. 2011;86(12): 1001-1006.

37. Hoeltge GA, Domen RE, Rybicki LA, Schaffer PA. Multiple red cell transfusions and alloimmunization: experience with 6996 antibodies detected in a total of 159,262 patients from 1985 to 1993. Arch Pathol Lab Med. 1995;119(1):42-45.

38. Aygun B, Padmanabhan S, Paley C, Chandrasekaran V. Clinical significance of RBC alloantibodies and autoantibodies in sickle cell patients who received transfusions. Transfusion. 2002;42(1):37-43.

39. Mueller-Eckhardt C, Kiefel V, Santoso S. Review and update of platelet alloantigen systems. Transf Med Rev. 1990;4(2):98-109.

40. Curtis BR, McFarland JG. Human Platelet Antigens - 2013. Vox Sang. 2014;106(2):93-102.

41. Storry JR, Castilho L, Daniels G, et al. International Society of Blood Transfusion Working Party on red cell immunogenetics and blood group terminology: Berlin report. Vox Sang. 2011;101(1):77-82.
42. Avent ND. Human erythrocyte antigen expression: its molecular bases. Br J Biomed Sci. 1997;54(1):16-37.

43. Mollison PL. Immunology of red cells. In: Klein HG, Anstee DJ, editors. Mollison's Blood Transfusion in Clinical Medicine. 11th ed. London: Blackwell; 2005:48-113.

44. O’Suoji C, Liem RI, Mack AK, Kingsberry P, Ramsey G, Thompson AA. Alloimmunization in sickle cell anemia in the era of extended red cell typing. Pediatr Blood Cancer. 2013;60(9):1487-1491.

45. Castro O, Sandler SG, Houston-Yu P, Rana S. Predicting the effect of transfusing only phenotype-matched RBCs to patients with sickle cell disease: theoretical and practical implications. Transfusion. 2002;42(6):684-690.

46. Wahl S, Quirolo KC. Current issues in blood transfusion for sickle cell disease. Curr Opin Pediatr. 2009;21(1):15-21.

47. Vichinsky EP. Current issues with blood transfusions in sickle cell disease. Semin Hematol. 2001;38(1 Suppl 1):14-22.

48. Afenyi-Annan A., Bandarenko N. Transfusion Practices for patients with sickle cell disease at a major academic medical center. Immunohematology 2006;22(3):103-107.

49. Reid ME, Rios M, Powell VI, Charles-Pierre D, Malavade M. DNA from blood samples can be used to genotype patients who have recently received a transfusion. Transfusion. 2000;40(1):48-53.

50. Legler TJ, Eber SW, Lakomek M, et al. Application of RHD and RHCE genotyping for correct blood group determination in chronically transfused patients. Transfusion. 1999;39(8):852-855.

51. Castilho L, Rios M, Bianco C, et al. DNA-based typing of blood groups for the management of multiply-transfused sickle cell disease patients. Transfusion. 2002;42(2):232-238.

52. Castilho L, Rios M, Pellegrino J Jr, O Saad S, F Costa F. Blood group genotyping facilitates transfusion of beta-thalassemia patients. J Clin Lab Anal. 2002;16(5):216-220.

53. Bakanay SM, Ozturk A, Ileri T, et al. Blood group genotyping in multitransfused patients. Transfus Apher Sci. 2013;48(2):257-261.

54. da Costa DC, Pellegrino J Jr, Guelsin GA, Ribeiro KA, Gilli SC, Castilho L. Molecular matching of red blood cells is superior to serological matching in sickle cell disease patients. Rev Bras Hematol Hemoter. 2013;35(1):35-38.

55. Wagner FF. Why do we use serological blood group phenotype determination in chronically transfused patients? Blood Transfus. 2014;12(1):1-2.

56. Denomme GA. Prospects for the provision of genotyped blood for transfusion. Br J Haematol. 2013;163(1):3-9.

57. Singleton BK, Green CA, Avent ND, et al. The presence of an RHD pseudogene containing a 37 base pair duplication and a nonsense mutation in most Africans with the RhD-negative blood group phenotype. Blood. 2000;95(1):12-18.

58. Castilho L. The value of DNA analysis for antigens in the Duffy blood group system. Transfusion. 2007;47(Suppl 1):28S-31S.

59. Storry JR, Reid ME, Fetics S, Huang CH. Mutations in GYPB exon 5 drive the S-s-U+(var) phenotype in persons of Africans descent: implications for transfusion. Transfusion. 2003;43(12):1738-1747.

60. Omoto R, Reid ME, Castilho L. Molecular analysis of GYPB in African Brazilians. Immunohematology. 2008;24(4):148-153.

61. Beiboer SH, Wieringa-Jelsma T, Maaskant-Van Wijk P, et al. Rapid genotyping of blood group antigens by multiplex polymerase chain reaction and DNA microarray hybridization. Transfusion. 2005;45(5): 667-679.

62. Curtis BR, McFarland JG. Detection and identification of platelet antibodies and antigens in the clinical laboratory. Immunohematology. 2009;25(3):125-135.

63. Kiefel V. The MAIPA assay and its applications in immunohaematology. Transfus Med. 1992;2(3):181-188.

64. Shibata Y, Juji T, Nishizawa Y, Sakamoto H, Ozawa N. Detection of platelet antibodies by a newly developed mixed agglutination with platelets. Vox Sang. 1981;41(1):25-31.

65. Bertrand G, Kaplan-Gouet C. Human platelet antigen genotyping and diagnosis of antiplatelet alloimmunization. In: Ness PM, Sloan SR, Moulds JA, editors. BeadChip Molecular Immunohematology. New York: Springer; 2011:73-81. 
66. Shafi H, Abumuhor I, Klapper E. How we incorporate molecular typing of donors and patients into our hospital transfusion service. Transfusion. 2014;54(5):1212-1219.

67. Sapatnekar S, Figueroa PI. How do we use molecular red blood cell antigen typing to supplement pretransfusion testing? Transfusion. 2014;54(6):1452-1458.
68. Hashmi G, Shariff T, Zhang Y, et al. Determination of 24 minor red blood cell antigens for more than 2,000 blood donors by high-throughput DNA analysis. Transfusion. 2007;47(4):736-747.

69. Tilley L, Grimsley S. Is Next Generation Sequencing the future of blood group testing? Transfus Apher Sci. 2014;50(2):183-188.

\section{Publish your work in this journal}

International Journal of Clinical Transfusion Medicine is an international, peer-reviewed, open access, online journal publishing clinicalexperimental, policy-making and evidence-based practices of all topics pertaining to clinical transfusion medicine. Original research, short reports, reviews, case reports and commentaries are invited.
The manuscript management system is completely online and includes a very quick and fair peer-review system, which is all easy to use. Visit http://www.dovepress.com/testimonials.php to read real quotes from published authors.

Submit your manuscript here: http://www.dovepress.com/international-journal-of-clinical-transfusion-medicine-journal 